\title{
Case-Based Reasoning for Eliciting the Evolution of Geospatial Objects
}

\author{
Joice Seleme Mota ${ }^{\mathrm{I}}$, Gilberto Câmara ${ }^{\mathrm{I}}$, Maria Isabel Sobral Escada ${ }^{\mathrm{I}}$, Olga Bittencourt ${ }^{\mathrm{I}}$, \\ Leila Maria Garcia Fonseca ${ }^{\mathrm{I}}$, and Lúbia Vinas ${ }^{\mathrm{I}}$, \\ ${ }^{\mathrm{I}} \mathrm{INPE}$ - National Institute for Space Research, \\ Av. dos Astronautas 1758, \\ I2227-OOI São José dos Campos, Brazil \\ \{joice, gilberto, isabel, olga, leila, lubia\}@dpi.inpe.br
}

\begin{abstract}
This paper proposes an automated approach for describing how geospatial objects evolve. We consider geospatial objects whose boundaries and properties change in the time, and refer to them as evolving objects. Our approach is to provide a set of rules that describe how objects change, referred to as rule-based evolution. We consider the case where we are given a series of snapshots, each of which contains the status of the objects at a given time. Given this data, we would like to extract the rules that describe how these objects changed. We use the technique of case-based reasoning (CBR) to extract the rules of object evolution, given a few representatives examples. The resulting rules are used to elicit the full history of all changes in these objects. This allows finding out how objects evolved, recovering their history. As an example of our proposed approach, we include a case study of how deforestation evolves in Brazilian Amazonia Tropical Forest.
\end{abstract}

\section{Introduction}

The computational modelling of geospatial information continues to be, after decades of research, a problem which defies a definitive solution. Since computer models assign humanconceived geographical entities to data types, matching geospatial data to types and classes has been the focus of intense research. Recently, there has been much interest on modelling and representation of geospatial objects whose properties change [I-5]. Such interest has a strong practical motivation. A new generation of mobile devices has enabled new forms of communication and spatial information processing. Remote sensing data is becoming widespread, and more and more images are available to describe changes in the landscape. As new data sources grow, we are overwhelmed with streams of data that provide information about change. 
Representing change in a GIS (Geographical Information System) is not only an issue of handling time-varying data. It also concerns how objects gain or lose their identity, how their properties change, which changes happen simultaneously, and what causes change. As Goodchild et al.[5] point out, the distinction between geospatial entities as continuous fields or discrete objects also applies in the temporal domain. In this paper, we deal with computational models for time-varying discrete geospatial entities. We refer to those as geospatial objects and distinguish two broad categories. The first category concerns objects whose position and extent change continuously, referred to as moving objects. The second type concerns objects bound to specific locations, but whose geometry, topology and properties change but at least part of its position is not altered. We refer to these as evolving objects, which arise in urban cadastre and in land cover change. For an alternative characterization of spatio-temporal objects, see Goodchild et al.[5].

This paper describes a computational model for evolving objects, which tracks changes that occurred during an object's lifetime. The proposed model aims to answer questions such as "What changes took place for each object?", "When did these changes occur?" and "How did the changes take place?". We aim to extract the history of an object from its creation to its disappearance, including references to other objects involved. Eliciting the history of each object helps us to understand the underlying causes of change. To be able to record the complete history of each object, we need a model that uses previous cases as well as knowledge obtained from an expert as the main sources of knowledge used to solve new problems. This leads to the subject of this paper. We propose a computational method that contains a set of rules that describe how geospatial objects evolve, based on a sample of existing cases. For this task we have used the Case-Based Reasoning (CBR) technique, which defines a set of rules that arise from knowledge about the application domain.

In what follows, we review previous work on section 2 and describe our proposal in Section 3. In section 4, we describe an experiment where we applied our method to a spatiotemporal study of deforestation evolution. This paper builds on previous work by the authors [6-8].

\section{Challenges in describing how spatial objects evolve}

In this section, we consider previous work on models for evolving objects and introduce the challenges in describing how these objects change. Evolving objects are typical of cadastral and land change applications. Computational models for describing such objects are also referred to as lifeline models. Lifeline models use three ideas: identity, life, and genealogy. Identity is the characteristic that distinguishes each object from others during all its life. Life is the time period from the object's creation until its elimination. Genealogy implies managing the changes that occur to an object has during its life. Hornsby and Egenhofer [9] stress the need to preserve an object's identity when its geometry, topology, or attributes change, a view supported by Grenon and Smith [3]. Consider parcels in an urban cadastre. A parcel can have its owner changed, be merged with another, or split into two. A possible 
approach is to describe an object's history based on operations such as creation, splitting and merging [9-IO]. However, these authors do not consider the problem of extracting evolution rules from the objects themselves. They consider objects of a single type. In this paper, we consider objects of different types and we provide ways to extract their evolution rules.

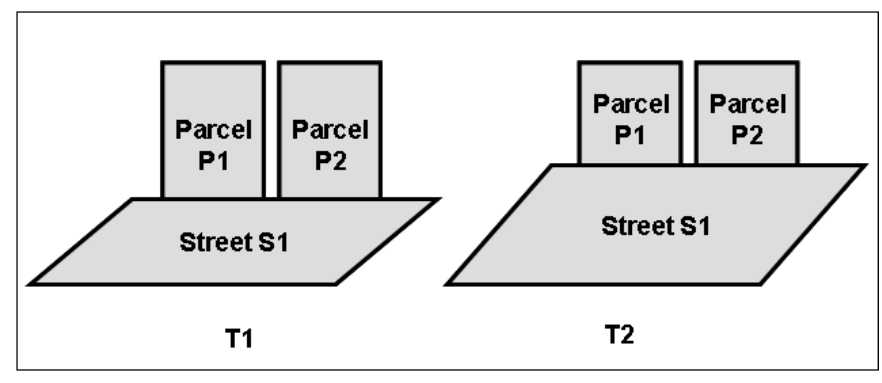

Fig. I. Example of objects evolution.

To take a simple motivational example, consider Figure I, where there are three objects: $\mathrm{S}_{\mathrm{I}}$ of type 'Street' and Pr and $\mathrm{P}_{2}$ of type 'Parcel'. Given the geometries of these objects at times $T_{I}$ and $T_{2}$, bow can we find out bow these objects evolved? To model this example, we need to consider different rules for spatial operations. Consider the case of the 'merge' geometric operation, which joins the geometries of two objects. When the objects have different types, merging two objects can produce different results. When the object types are 'Street' and 'Parcel', there should be different rules for the result of the merging two objects. One possible set of rules is: (a) "merging two Parcels results in a Parcel"; (b) "merging a Street with a Parcel results in expanding the Street".

As a second example, consider how the internal and external borders of Brazil changed, as shown in Figure 2. Each polygon in Figure 2 is a Brazilian state. The Brazilian borders have changed significantly since the $18^{\text {th }}$ Century, both because of internal division (creation of new states from existing ones) and inclusion of external areas (through international treaties). Suppose we want to devise a procedure that, given the snapshots shown in Figure 2, tries to extract the history of Brazil's internal and external borders. Such method would have to distinguish at least three data types ('Country', 'State', 'ExternalArea') and would need a set of type-dependent rules for object merging and splitting. As a first guess, this set would have these rules:

RI. Splitting an existing State produces two States: a new State and the existing State with a smaller area.

R2. An existing State can be converted into a new State with the same borders.

R3. Merging a State with an existing State produces a State with larger area. The new area is assigned to an existing State.

R4. Merging a Country with an External Area produces a Country with larger area. The new area is assigned to an existing State. 
R5. Splitting a State from a Country produces a Country with smaller area and a new part of the External Area.

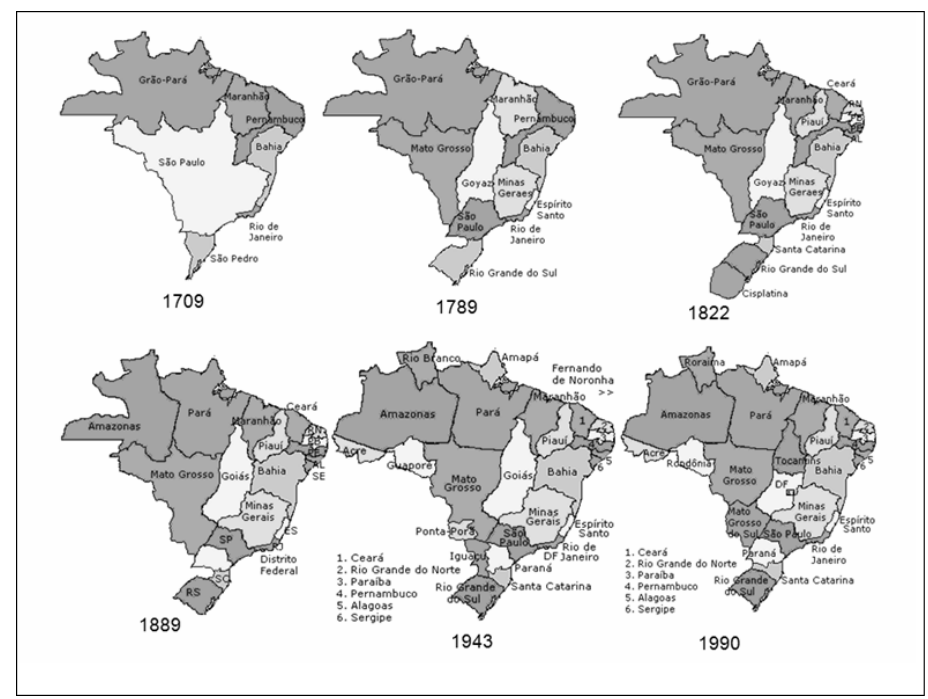

Fig. 2. Evolution of internal and external borders of Brazil from I709 to 1990.

These rules are not the only possible set. They may be able to rebuild a believable history of the Brazilian states, but may fail to be historically accurate. Given a set of snapshots which show that state of spatial objects in different times, we are not always able to remake their precise history. However, often snapshots are all we have, and we need to devise ways to make a likely guess about the objects' evolution.

These examples and similar cases lead us to propose the idea of rule-based evolution of typed geospatial objects. Our view of types comes from Computer Science, where types are tools for expressing abstractions in a computer language [II]. On a theoretical level, a type is a set of elements in a mathematical domain that satisfy certain restrictions. A typed object is an object whose evolution is subject to constraints that are specific to its type. Thus, in the Brazilian borders example, for objects of type 'Country' and those of type 'State' we need different rules to describe their evolution. Models in which objects have different types and evolution cases are richer and more powerful than typeless ones.

\section{Extracting the Evolution Rules using Case-Based Reasoning}

In this section, we describe the use of Case-Based Reasoning (CBR) to extract the evolution rules for a set of geospatial objects. CBR is a method for problem solving that relies on previously used solutions to solve similar problems [I2]. In contrast to techniques that rely solely on general knowledge of a problem domain, CBR is able to use the specific knowledge of previously experienced, concrete problems [13]. This is a recurring technique in humanproblem solving. To solve a new problem, we recall how we handled a similar one in the past and try to reuse it. A new problem is solved by finding a similar past case and reusing it in the new problem. CBR is an incremental technique, since experience learned in a case is applied for solving future problems. 
Traditional rule-based inferences have no memory of previous cases, and use the same set of rules for solving all problems. Instead of doing inferences using a large group of rules, a CBR-based software keeps track of previous cases. When confronted with a new problem, it tries to adapt rules that were useful in similar cases, thus increasing continually its knowledge base [13]. The similarity between two problems can often ensure the interpretation adopted for a previous case can also adopted for the new one.

There are two types of CBR implementations: automatic procedures and information recovery methods [I2]. Automatic systems solve the problem in an autonomous way and provide methods to evaluate the results of their decisions. Information recovery methods use human experts to set up the problem-solving rules based on well-known examples. These rules are then used to perform the desired task. The current work uses a CBR system of the second type. Following [I3], our proposed CBR technique has the following main steps: I) Select a set of exemplary cases in the database; 2) Use these cases to set up a set of evolution rules with the help of a domain expert; 3 ) Test the proposed solution and, if necessary, revise it; and 4) Store the experience represented in the current set of rules for future reuse. The steps to model and to represent how spatiotemporal objects evolve (shown in Figure 3) are:

I. Retrieval of snapshots of the area that contains a set of geospatial objects whose bistory we want to describe.

2. Selection of a subset of this data that allows the buman expert to find out the different types of geospatial objects and set up their evolution rules.

3. Represent these evolution rules using $C B R$.

4. Recover all objects from the database and compute their bistory based on the evolution rules.

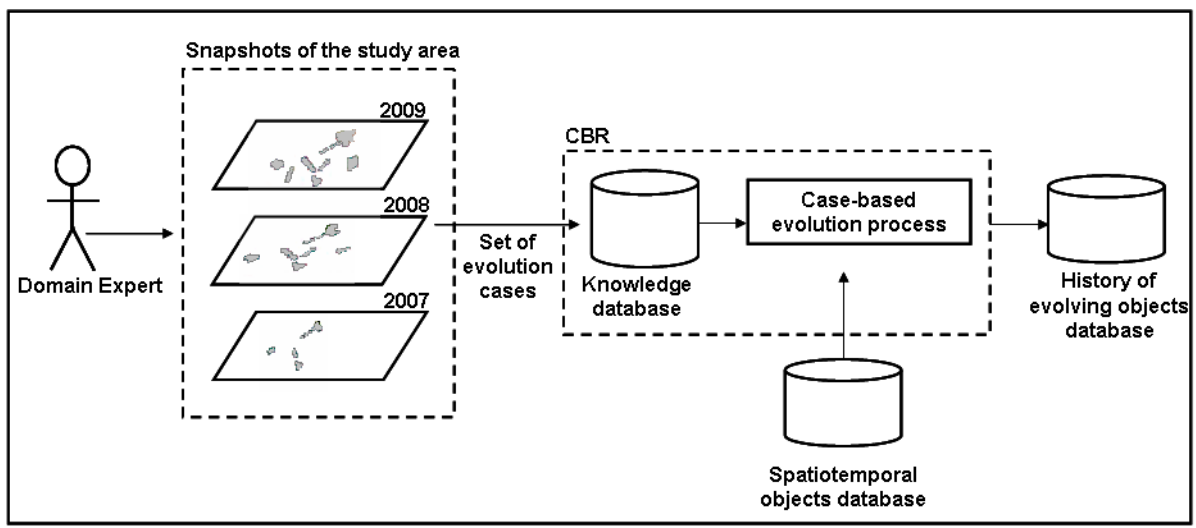

Fig. 3. General view of CBR method for eliciting geospatial objects evolution.

The domain expert defines two types of rules to characterize the objects' evolution: description rules and evolution rules. The description rules define the types of geospatial objects. The evolution rules define how objects evolve under spatial operations such as 'split' and 'merge'. The expert defines the description rules considering the objects' properties and their 
spatial relationship, including topological predicates such as 'cross', 'close to' and 'touch'. Consider Figure 4, where some prototypical land change objects are portrayed. Figure 4(a) shows three objects at time $\mathrm{T}_{1}$. At time $\mathrm{T}_{2}$, three new objects appear as shown in Figure 4(b). After applying the description and evolution rules described below, the resulting objects are shown in Figure 4(c).

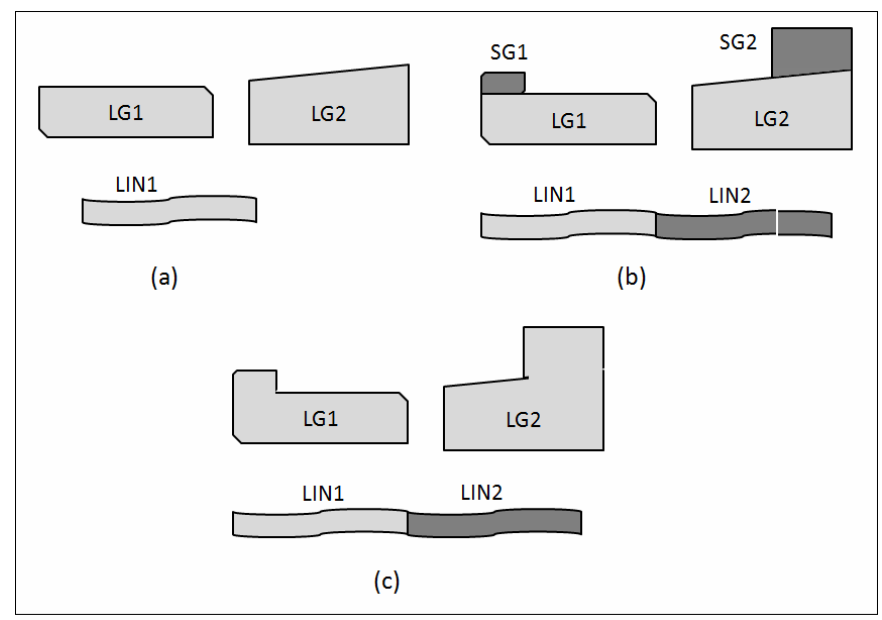

Fig. 4. Evolution of prototypical land change objects: (a) Time $\mathrm{T}_{\mathrm{I}}$; (b) Time $\mathrm{T}_{2}$ before application of description rules; (c) Time $\mathrm{T} 2$ after application of evolution rules.

In this example, the description rules define three types of objects: LargeGeometric $(L G)$, Linear (LIN) and Small Geometric (SG), according to the following rules:

DRI. An object with perimeter/area ratio smaller than Io bectares $^{-I}$ is a Linear object.

DR2. An object with perimeter/area ratio greater than Io bectares ${ }^{-1}$ and whose area is less than 50 bectares is a Small Geometric object.

$D R_{3}$. An object with perimeter/area ratio greater than $I_{0}$ bectares $^{-I}$ and whose area is more than 50 bectares is a Large Geometric object.

These rules allow us to identify the objects in Figure 4, as shown in the labels assigned to each object. For this case, a possible set of evolution rules would be:

ERI. A Small Geometric object that touches a Large Geometric object is merged with the Large Geometric object.

ER2. Two adjacent Small Geometric objects are merged.

ER3. Two Linear objects that are adjacent are not merged.

Applying these rules, the SmallGeometric objects shown in Figure 4(b) are merged with the adjacent LargeGeometric objects, thus resulting in a spatial expansion of the latter. This example shows the need for a system that is able to represent the description and evolution 
rules and to apply them to extract the history of a set of objects. This system architecture is described in the next section.

\section{CBR-based Geospatial Object History Extractor}

This section describes the architecture of a geospatial history extractor based on Case-Based Reasoning (CBR) technique. A CBR system stores knowledge as a set of cases. Each case contains data about a specific episode, with its description and the context in which it can be used. The contents of each case include a set of rules set up by the domain expert. Among the several existent techniques for knowledge acquisition for CBR, we used unstructured interviews, where the information is obtained through direct conversation with the specialist. In these interviews, he gives his perspective of the problem, and a computer specialist records these cases. The expert elicits the knowledge domain in two steps:

I. Describing the objects in their environment (description rules).

2. Analyzing this outcome of spatial operations between the objects (evolution rules).

After the expert produces the rules, the CBR system stores a set of rules for each case, as shown in Figure 5 .

The knowledge base consists of a series of cases, indexed by the object's attributes. Based on the problem's description, the indexes point out which attributes should be compared, finding out the case that can be useful for the solution. Each attribute receives a weight (among $\mathrm{O}$ and $\mathrm{I}$ ) according to their degree of importance in the solution of the case. In our model we built the indexes using an explanation-based technique, where the specialist points out which attributes are relevant for the solution of the problem. Figure 5 shows the indexes for the cases that described the problem described in Figure 4. The indexes for the description rules are area and perimeter/area ratio; the indexes for evolution cases are the objects types and their spatial relationship. 


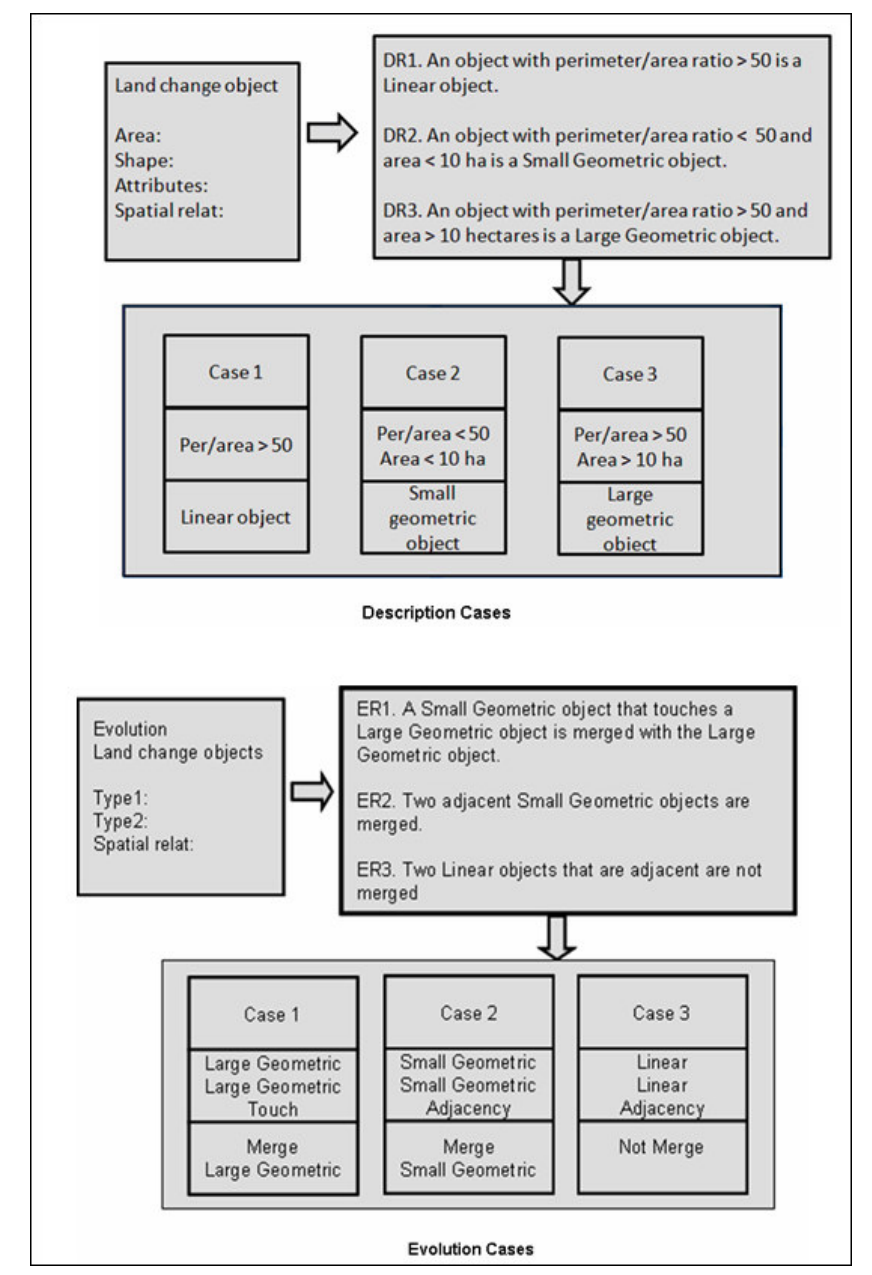

Fig. 5. Example of case construction based on expert rules

After creating and indexing the knowledge base, we can then create the history of all objects. Each object is considered as a new problem and processed separately in two phases. Processing starts by taking the objects from the Geospatial Objects Database that contains snapshots of the geospatial objects at different periods of time. For the example shown in Figure 2 (evolution of Brazil's borders), the database would contain six snapshots for the years I709, 1789, I822, I889, I943 and I990. The CBR system starts at the earliest snapshot. For each object in each snapshot, the CBR tries to find out its type based on the Description Rules, defined by a domain expert. The CBR system measures the similarity between each case stored in the database and the new object, according to their attribute values. Expressed as a real number between 0.0 (no similarity) and I (equality), similarity is calculated for each case in the database according to the attribute values. The software recovers the best match, shows it to the expert for confirmation, and stores the confirmed solutions in the Typed Geospatial Objects Database. After processing all the information from the first snapshot, the system recovers all objects from the next snapshot in the Geospatial Objects Database. It describes them according to the Description Rules and stores them in the Typed Geospatial Objects Database. 
The second phase of the CBR-based system takes the objects from two consecutive snapshots of the Typed Geospatial Objects Database to describe their evolution. The specialist verifies if the objects are neighbors and the system compares the objects from the two consecutive snapshots according to the rules of the Evolution Cases Database. These rules consider the objects' spatial relationships to find out if two objects should be merged in agreement with their description. The system creates the history of each object and stores it in the History Objects Database. The attributes of the History Objects Database are:

I. New Object: the new identification for the object created.

2. FatherObjectI: identification of the first object that generated the new.

3. YearObject: : year of the first object that generated the new.

4. FatherObject2: identification of the second object that generated the new.

5. YearObject2: year of the second object that generated the new.

6. Result: the new description for the new object.

7. Year result: the year in which the new object was created.

8. New area: the area of the new object given by the sum of the areas of the objects that were merged.

These attributes keep the origin of the new object allowing the recovery of its history. Considering that the snapshots are stored in increasing temporal order, taking time as a sequence $T=[14]$ the evolving process can be described in the following steps:

I. Let $\mathrm{T}=\mathrm{I}$.

2. Take the objects from time $T$ from the Geospatial Objects Database. Describe these objects according to the Description Cases Database. Store the results in the Typed Geospatial Objects Database.

3. Take the objects from time $\mathrm{T}+\mathrm{I}$ from the Geospatial Objects Database. Describe these objects according to the Description Cases Database. Store the results in the Typed Geospatial Objects Database.

4. Compare the objects of times $\mathrm{T}$ and $\mathrm{T}+\mathrm{I}$ using the Evolution Cases Database. Evolve the objects if possible. Store the results in a History Objects Database.

5. If there are further snapshots in the Geospatial Objects Database, make $T=T+I$ and go to step 2 above. Otherwise, exit the program. 
The example shown in Figure 5 is a simple one, where the cases are mutually exclusive and simple ad-hoc rules could solve the problem without resorting to CBR. Many cases are not mutually exclusive and require reasoning techniques which are more complex. To better explain the possible uses of the proposed technique, we present a case study using real data in the next section.

\section{Land change objects in Brazilian Amazonia: a case study}

This section presents a case study about extraction of the history of geospatial objects associated to deforestation areas in the Brazilian Amazonia rainforest. The motivation is the work carried out by the National Institute for Space Research (INPE). Using remote sensing images, INPE makes yearly assessments of the deforestation in Amazonia region. INPE's data show that around 250,000 km² of forest were cut in Amazonia from 1995 to 2007 [I5].

Given the extent of deforestation in Amazonia, it is important to figure out the agents of deforestation. We need to assess the role and the spatial organization of the different agents involved in land change. Our idea is to associate each land change patch, detected in a remote sensing image, to one of the agents of change. Extensive fieldwork points out the different agents involved in land use change (small-scale farmers, large plantations, cattle ranchers) can be distinguished by their different spatial patterns of land use [I6] [8]. These patterns evolve in time; new small rural settlements emerge and large farms increase their agricultural area at the expense of the forest. Farmers also buy land from small settlers to increase their property for large-scale agriculture and extensive cattle ranching. Therefore, CBR system will aim to distinguish land change objects based on their shapes and spatial arrangements.

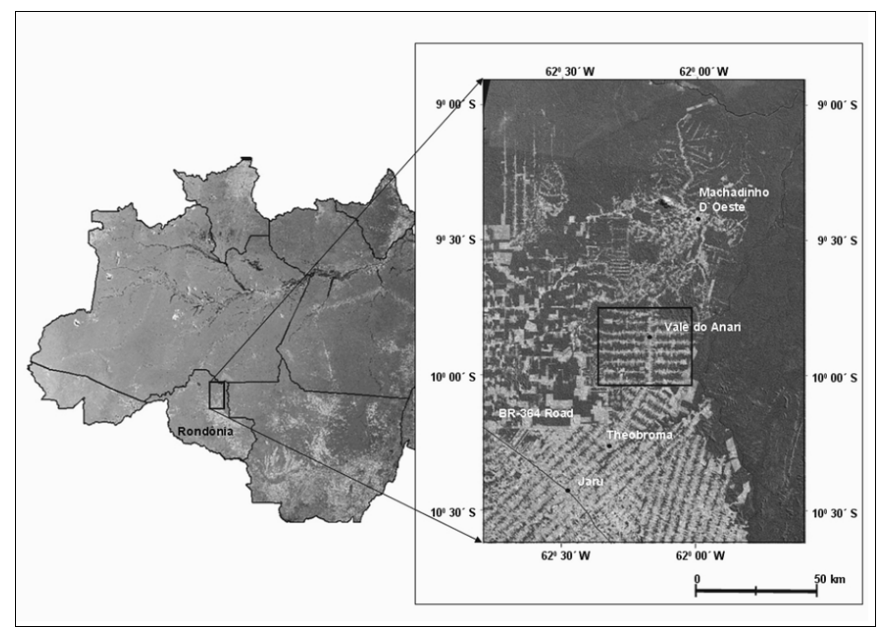

Fig. 6. Location of the study area. The Brazilian Amazonia is on the left, and the Vale do Anari area in the state of Rondônia is on the right. Light-coloured areas indicate deforestation.

We selected a government-planned rural settlement called Vale do Anari, located in Rondônia State, in Brazilian Amazonia Tropical Forest. This settlement was established by INCRA (Colonization and Land Reform National Institute), in 1982, with lots of 
approximately 50 ha (see Figure 6). The third author had carried out fieldwork on the area [I7-I8]. In their work, Silva et al [8] used a decision-tree classifier to describe shapes found in land use maps extracted from remote sensing images. They associated these shape descriptions to different types of social agents involved in land use change. Silva et al [8] work did not find out how individual objects evolved, but presented their results comparing the overall types of objects found in each snapshot. They classified deforestation patterns as Linear (LIN), Irregular (IRR) and Geometric (GEO) in Vale do Anari region [8]. These objects associated a deforestation patterns were the input of our system. In our study, we distinguish three types of land change objects: Small Lot (LOTS), Along Road Occupation (AR) and Concentration Areas (CON). The main characteristics of those objects are:

I. Along Road Occupation: Small settlement household colonists living on subsistence agriculture or small cattle ranching. Their spatial patterns show up as linear patterns following planned roads built during earlier stages of colonization.

2. Small Lot: Small household colonists associated to settlement schemes living on subsistence agriculture or small cattle ranching. Their spatial patterns show up as irregular clearings near roads, following parcels defined by the planned settlement.

3. Concentration: Medium to large farmers, associated to cattle ranches larger than 50 ha. This pattern results from the selling of several 50 ha lots to a farmer aiming to enlarge his property. Their spatial patterns is geometric, close to roads or population nuclei.

The Description Rules (DR) for deforestation objects in our case study are:

DRI. "A geometric spatial pattern is an object of type land concentration".

DR2. "An irregularly shaped pattern that touches a road is an object of type along road occupation".

$D R_{3}$. "An irregular spatial pattern doesn't touch a road is an object of type small lot".

$D R 4$. "A linear spatial pattern that touches a road is an object of type along road occupation".

DR5. "A linear spatial pattern that doesn't touch a road is an object of type small lot".

A subset of the deforestation objects in the Vale do Anari is shown in Figure 7. The sequence starts with objects representing I982-I985 deforestation on the right side. The next set of deforestation objects represents new deforested areas detected during the 1982I 985 period and so forth. These three year snapshot show how deforestation occurred; the objects' labelling was confirmed by experts on deforestation domain. On the left side of Figure 7 the deforestation objects detected in the intervals of three years are shown and linked to an attribute table by an identification number. 


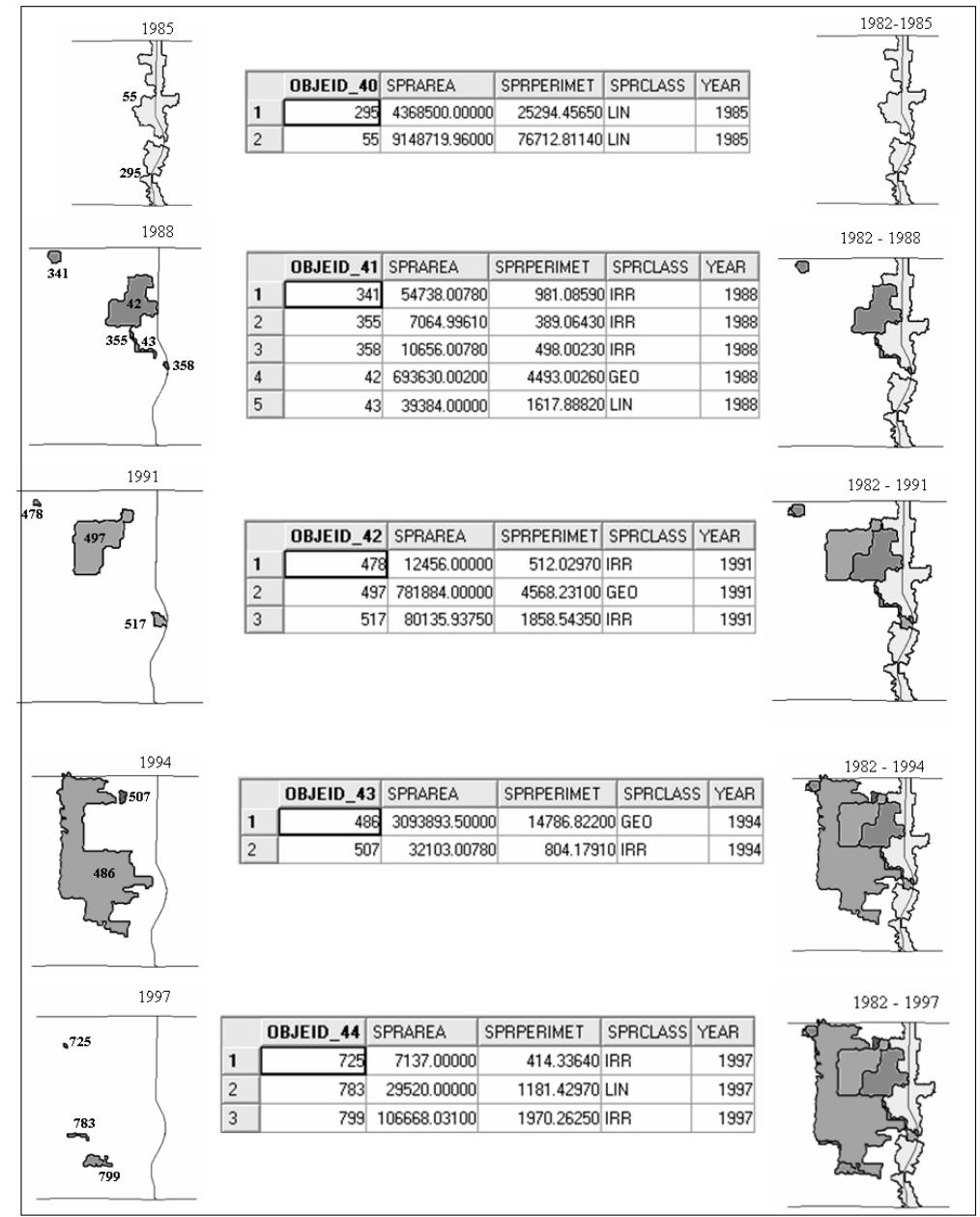

Fig. 7. Sequence of deforestation objects.

The next step is defining the evolution rules. These rules depend on the object's type and its relation with adjacent objects. An object of type along road occupation does not evolve, since it signals the start of the occupation. When two small lots touch each other, they are merged into a new small lot. When a concentration touches a concentration or a small lot, they are merged and the result is a new concentration. A small lot with area greater than 50 ha represents the results of small lots that were merged. If a concentration touches a small lot with area greater than 50 ha, it doesn't evolve. The evolution rules are:

ERI - "Two adjacent land concentration are merged into a land concentration".

$E R 2$ - "An object of type along road occupation is not merged with other objects".

$E R_{3}$ - "Two adjacent small lots are merged into a small lot".

ER4 - "A small lot with area $<50$ ba adjacent to a land concentration is merged with it and the result is a land concentration".

$E_{5}-$ "A small lot with area $>=50 b a$ and adjacent to a land concentration is not merged with other object." 
The CBR system builds the Description Cases Database using the description rules and the Evolution Cases Database using the evolution rules. Then, it considers all deforestation objects using the procedure described in Section 4 above. For each new object, it looks for a similar case in Description Cases Database to define its type. The next step is applying the evolution rules. Given an object's type and spatial arrangements, the CBR software looks for similar cases in the Evolution Cases Database. Based on these cases, it establishes the history of each object, including the originating objects (if the new objects results from a merge operation). The results produced for a sample of the deforestation objects are presented in Figure 8.

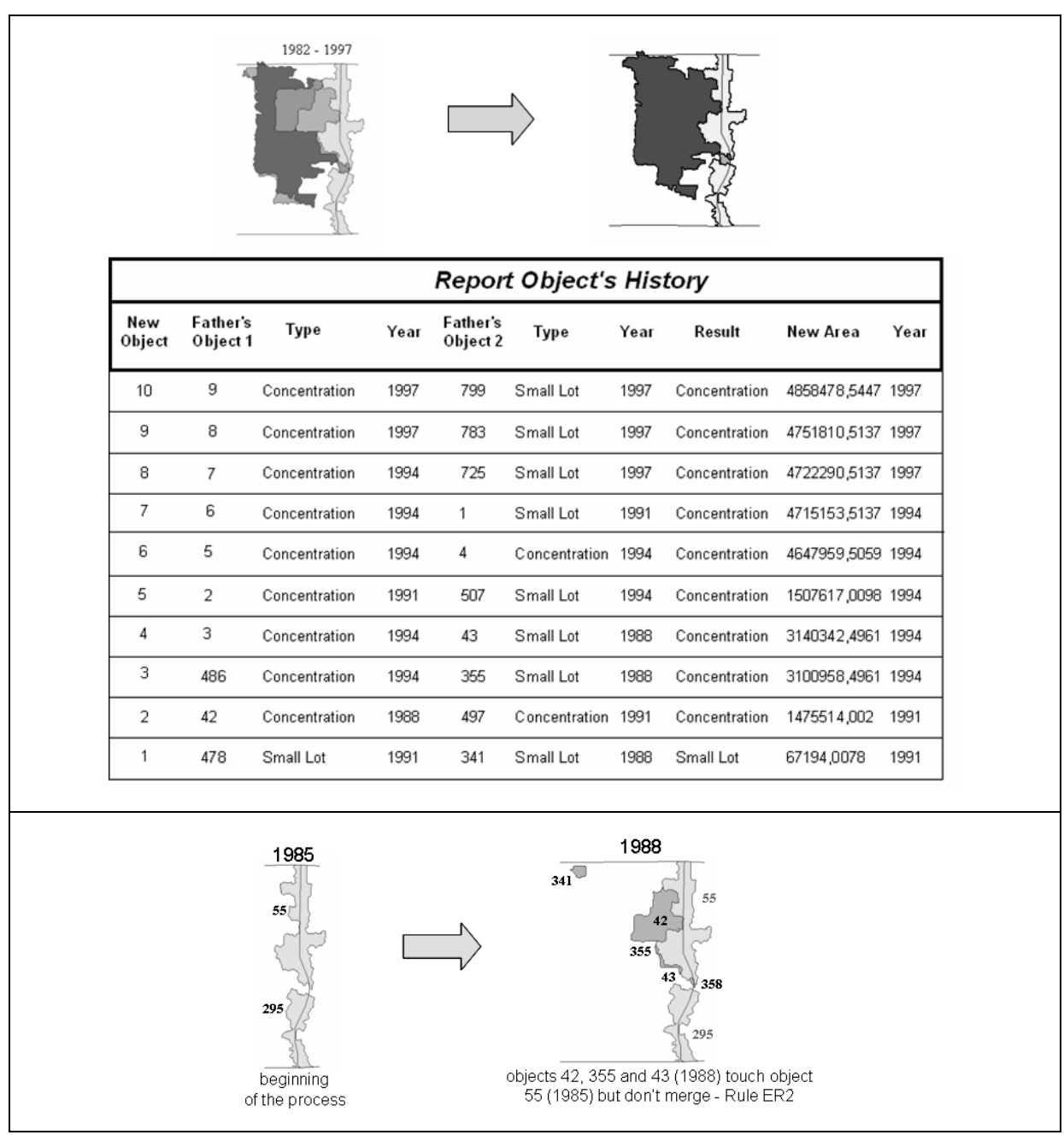




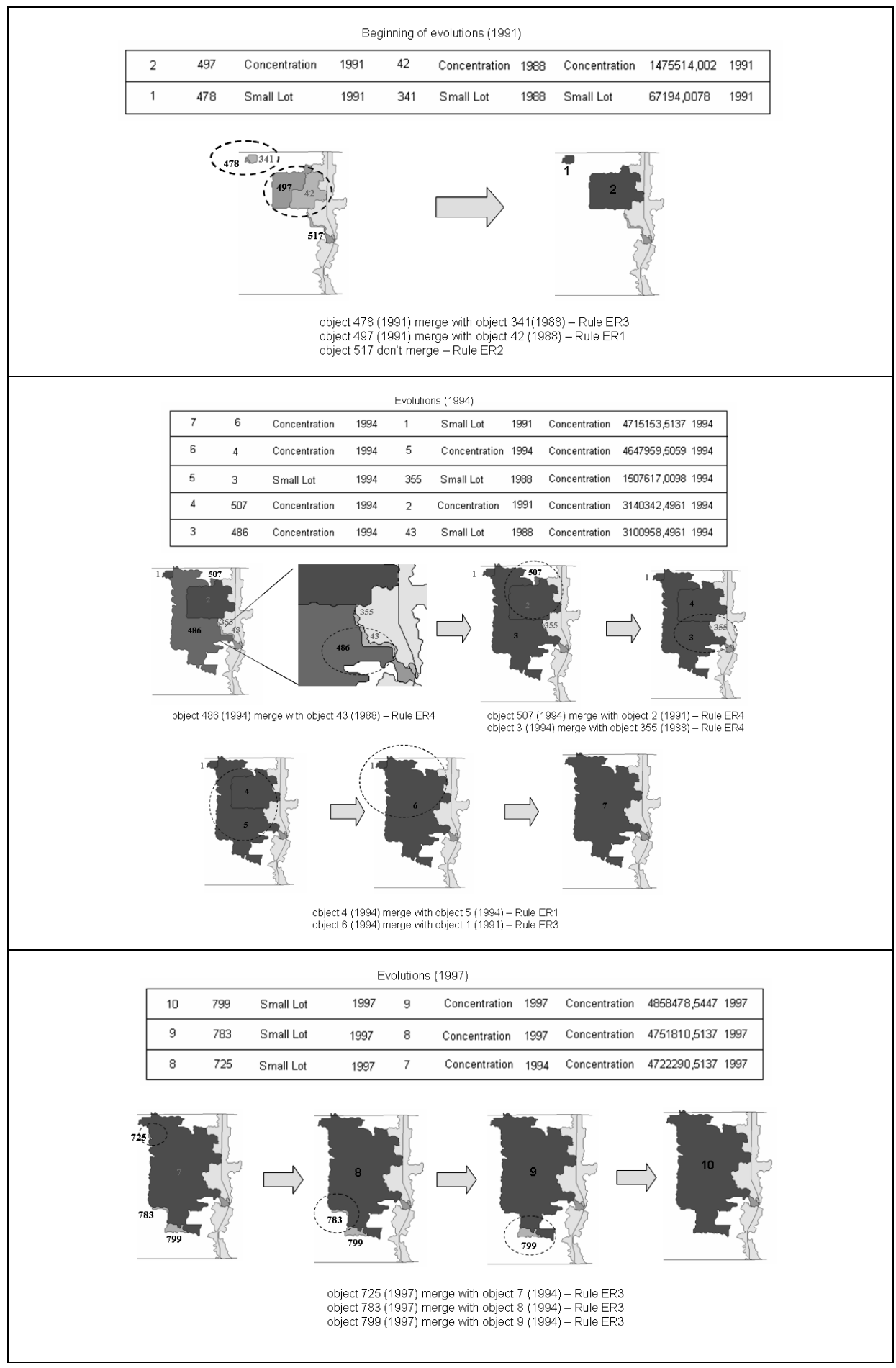

Fig. 8. Example of the history of deforestation objects.

The report of the CBR system shows how deforestation objects evolved. Until I99I, no objects evolved due to rule ER2: "An object of type along road occupation is not merged with other objects". In I99I, the object 478 merged with the object 34 I following rule ER 3 ("Two adjacent small lot objects are merged and the new object is a small lot") and the result is the object I. Also in I99I, object 497 merges with object 42 according to rule ERI, ("Two adjacent land concentration objects are merged and the new object is a land concentration"), creating 
object 2. In 1994, land concentration object 486 appears and merges with object 43 following rule $\mathrm{ER}_{4}$ ("A small lot with area $<50$ ba adjacent to a land concentration object is merged with it and the result is a land concentration object”.), creating object 3. In the same year, object 2 merges with object 517 again following rule ER 4 , creating object 4 . In I994 object 3 merges with object 355, following rule ER4, creating object 5. Still I994, object 4 merges with object 5, following rule ERI, creating object I, that is again expanded, producing object 7 , which merges with object I. In I997, object 7 merges with objects 725 , creating object 8 . Then it merges with object 783 , creating object 9, and finally merges with object 799 , producing object Io. The CBR system was thus able to show how land concentration occurred in the region, showing that the government plan for settling many colonists in the area has been largely frustrated [17]. The process of land concentration in the Vale do Anari settlement described by the CBR system matches what was noted in the interviews performed during fieldwork [I7].

\section{Conclusions}

In this paper, we dealt with evolving objects. We are interested in cases where the simple rules of merging and splitting are not enough to describe their evolution, since such evolution depends on the object's types. We propose a method that uses previous cases as well as knowledge elicited from a specialist as the main sources of knowledge used to solve new problems. A contribution of our research is the definition a case-based reasoning (CBR) method to describe the object's type and find out how geospatial objects evolve. Experimental results for the Brazilian Amazonia Tropical Forest corroborate the effectiveness of our proposal. The approach of using typed geospatial objects and evolution rules contributes to solve the problem of automatically modelling and describing the history of evolving geospatial objects.

Use of the CBR software for describing object evolution follows form the work of Silva et al. [I3] that developed a method for distinguishing patterns of land use change based on their shapes in static timestamps. Their work did not discuss how spatial patterns evolve in time. The current work addresses the problem of tracking changes during an object's lifetime, based on type-specific evolution rules. In our experiments using case-based reasoning (CBR), we were able to obtain the rules for object evolution and to describe how geospatial objects evolve. The CBR technique proved to be a simple and useful approach to set up the rules for land change trajectories.

In our application domain, CBR presented a satisfactory result, since the knowledge base had only a few cases, which were presented to the expert in an organized way. When there are many data types and different cases, the knowledge base should be generated carefully to avoid conflicting and inconsistent interpretations. Additionally, despite advanced techniques for case indexing and retrieval (neural networks, genetic algorithms), a knowledge base with many cases can have a slow and bad performance. In such cases, the CBR software needs to include adaptation and learning techniques, which also detect inconsistencies in the rules. In 
this case, the rules would be changed according to the expert's reaction to examples being presented to him. Adaptation and learning are complex and error-prone techniques that, if not done properly, may result in further inconsistencies in the knowledge base. Therefore, many CBR softwares do not provide an adaptation and learning facility. They simply recover the most similar case and make the solution available for the specialist to determine if it solves his matching problem.

Our experience shows that CBR-based techniques are useful and simple to set up for recovering the history of evolving geospatial objects when there are few types and clear-cut rules for object description and evolution. When there are many types and the evolution rules are complex, the CBR software needs to be carefully designed, and should include a learning phase and techniques for detecting inconsistencies and conflicts. In our work, we consider the CBR software we designed in promising enough; so we plan to extend these CBR techniques for improving the study of land cover change evolution in the Brazilian Amazonia region.

\section{References}

I. Frank, A., Ontology for Spatio-temporal Databases, in Spatio-Temporal Databases: The Chorocbronos Approach (Lecture Notes in Computer Science 2520), M. Koubarakis and T. Sellis, Editors. 2003, Springer-Verlag: Berlin. p. 9-78.

2. Worboys, M., Event-oriented approaches to geographic phenomena. International Journal of Geographical Information Science, 2005. I9(I): p. I-28.

3. Grenon, P. and B. Smith, SNAP and SPAN: Towards Dynamic Spatial Ontology. Spatial Cognition \& Computation, 2003.4(I): p. 69-IO4.

4. Galton, A., Fields and Objects in Space, Time, and Space-time. Spatial Cognition and Computation, 2004.4(I): p. 39-68.

5. Goodchild, M.F., M. Yuan, and T.J. Cova, Towards a general theory of geograpbic representation in GIS. International Journal of Geographical Information Science, 2007. 21(3): p. 239-26o.

6. Bittencourt, O., et al. Rule-based evolution of typed spatiotemporal objects. in VIII Brazilian Symposium in Geoinformatics, GeoInfo2007. 2007. Campos do Jordão, Brazil.

7. Mota, J.S., et al. Applying case-based reasoning in the evolution of deforestation patterns in the Brazilian Amazonia. . in Proceedings of the 2008 ACM Symposium on Applied Computing (Fortaleza, Ceara, Brazil, March I6 - 20, 2008). SAC'08. . 2008. ACM, New York, NY

8. Silva, M.P.S., et al., Remote Sensing Image Mining: Detecting Agents of Land Use Change in Tropical Forest Areas. International Journal of Remote Sensing, 2008. 29: p. 4803-4822.

9. Hornsby, K. and M. Egenhofer, Identity-Based Change: A Foundation for Spatio-Temporal Knowledge Representation. International Journal of Geographical Information Science, 2000. I4(3): p. 207-224. 
Io. Medak, D., Lifestyles, in Life and Motion of Socio-Economic Units. ESF Series, A. Frank, J. Raper, and J.-P. Cheylan, Editors. 200I, Taylor \& Francis: London.

II. Cardelli, L. and P. Wegner, On Understanding Type, Data Abstraction, and Polymorphism. ACM Computing Surveys, I985. I7(4): p. 47I-552.

12. Kolodner, J.L. and M.Y. Jona, Case-based reasoning: An overview. 1991: Technical Report I5 (Northwestern University, June).

13. Aamodt, A. and E. Plaza, Case-based reasoning: foundational issues, methodological variations and system approaches. . AI Communications, I994· 7(I): P. 39-59.

I4. Amazonica, A., et al.

15. INPE, Monitoramento da Floresta Amazônica Brasileira por Satélite (Monitoring the Brazilian Amazon Forest by Satellite). 2005, Report (São José dos Campos: INPE).

I6. Lambin, E.F., H.J. Geist, and E. Lepers, Dynamics of land-use and land-cover change in Tropical Regions. Annual Review of Environment and Resources, 2003. 28: p. 205-24I.

17. Escada, M.I.S., Evolução de Padrões da Terra na Região Centro-Norte de Rondônia. 2003, Instituto Nacional de Pesquisas Espaciais: São José dos Campos. p. I64 p.

I8. Souza, L.S., M.I.S. Escada, and P. Verbug, Quantifying deforestation and secondary forest determinants for different spatial extents in an Amazonian colonization frontier (Rondonia). Applied Geography (Sevenoaks), in press. 